\title{
New Web-Based Access to Nuclear Structure Datasets
}

\author{
D.F. Winchell \\ National Nuclear Data Center, Brookhaven National Laboratory, Upton, NY 11973 USA
}

\begin{abstract}
As part of an effort to migrate the NNDC databases to a relational platform, a new web interface has been developed for the dissemination of the nuclear structure datasets stored in the Evaluated Nuclear Structure Data File and Experimental Unevaluated Nuclear Data List.
\end{abstract}

\section{INTRODUCTION}

Nuclear structure information in the Evaluated Nuclear Structure Data File (ENSDF) and Experimental Unevaluated Nuclear Data List (XUNDL) databases is organized by dataset. Each dataset contains evaluated (ENSDF) or compiled (XUNDL) nuclear structure information from reaction or decay data. Contributions for ENSDF come from the international network of Nuclear Structure and Decay Data evaluators ${ }^{1}$. XUNDL was created in 1998, and contains compiled data from recent nuclear structure papers. XUNDL datasets are compiled primarily by the Nuclear Data Group at McMaster University ${ }^{2}$. Each dataset conforms to the standard ENSDF format [1], which permits further processing by a large base of existing programs. As of September 24, 2004, ENSDF contains 15250 datasets for 2920 nuclides, and XUNDL contains 1326 datasets for 916 nuclides.

One of the primary goals in developing a new database structure and web interface is to provide fast and flexible access to the data. A two-step process is used to retrieve data. First, the user identifies datasets of interest using various search criteria. Second, specific datasets can be chosen from the resulting list for display or download. For most of the search options, one set of criteria can be used to look for datasets in both the ENSDF or XUNDL databases, or searches can be restricted to just one of the databases.

Many of the search features in this implementation had been available via a telnet interface, but were not previously available on the web.

1 http: //www-nds.iaea.org/nsdd:html

2 http://physwww.physics.mcmaster.ca/ balraj/

\section{SELECTION MODES}

Most of the selection criteria used in the ENSDF/XUNDL web application are derived from the IDENT record (the first line) of the dataset. On the main page (http: //www.nndc.bnl.gov/ensdf/) the user is given a choice to search by nuclide, decay, or reaction properties, or to browse the datasets by mass or charge. In addition, separate indexes for the ENSDF and XUNDL databases are available. Providing a variety of entry points into the database allows the user to retrieve information in a way that best serves his or her needs.

\section{Search by nuclide}

This page allows the user to search by specific nuclide, or by mass or charge number. The latter two may be given as ranges. In place of charge number, element symbols can be used (i.e., Am-Fm or 95-100 are both valid for this field). This page also allows the user to specify dataset types for inclusion (Adopted, Reaction, Decay, Comments, or Reference), in addition to the database selection mentioned earlier.

Fig. 1 shows an example of this form, with both databases and all dataset types selected. The quantity " $160 \mathrm{~W}$ " is specified in the nuclide input field; when the corresponding "Search" button is pressed, a list of matching datasets will be retrieved.

A common feature found on most of the ENSDF/XUNDL pages is the small set of navigational links seen at the bottom of the figure. This allows the user quick access back to the "top-level" web pages. 


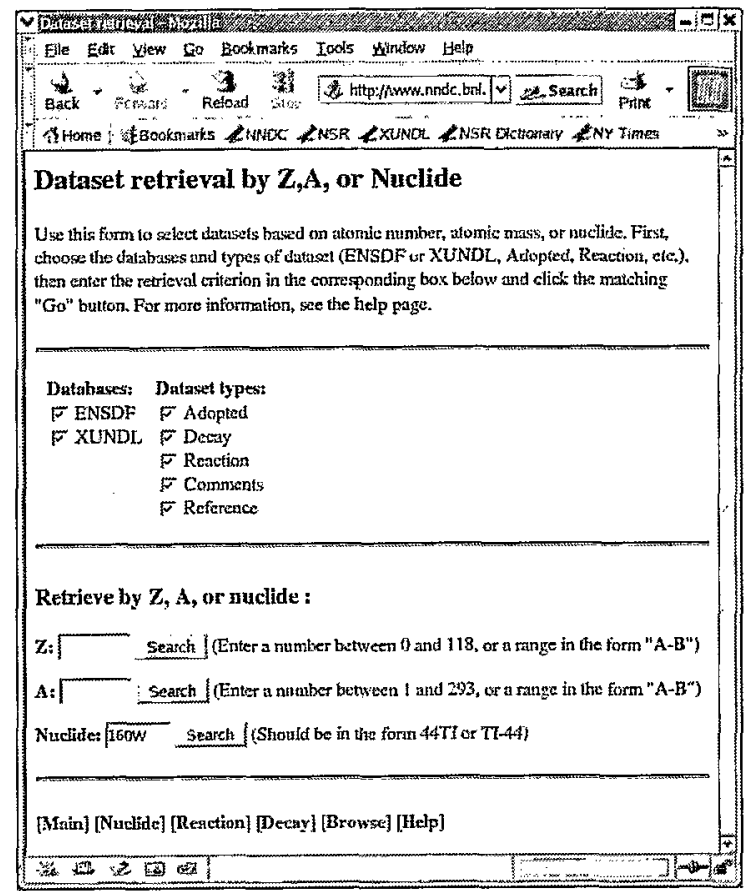

FIGURE 1. Input form for "Query by nuclide".

\section{Search by reaction and decay quantities}

For reaction datasets, there are typically one or more specific reactions listed in the DSW field of the IDENT record. This search page provides searches on quantities extracted from those reaction strings. These quantities include target, incident and outgoing particles, and residual nucleus. In addition, certain reaction "types" such as alpha-transfer, Coulomb excitation, etc., can be used as search criteria.

In decay datasets, the decay type and parent nuclide are listed in the DSID field. Either or both of these quantities can be used to retrieve datasets.

For both reaction and decay searches, the user can specify a range of charge and mass values. Fig. 2 shows a decay search, set to look for datasets in the mass range $100-150$ corresponding to ${ }^{252} \mathrm{Cf}$ spontaneous fission.

\section{Browse}

As an alternate to forms-based searching, a simple browse interface to the datasets is provided. Users are given lists of elements and masses. Choosing an element or mass brings up a list of matching nuclides, and links for retrieving datasets from ENSDF or XUNDL. This gives access to all the datasets without going through forms.

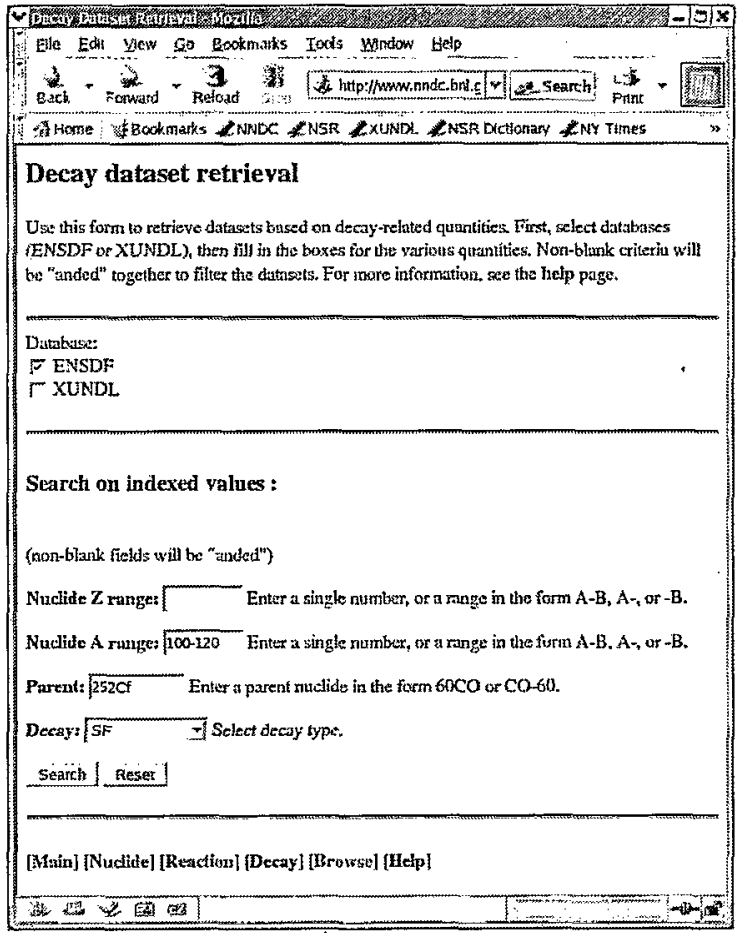

FIGURE 2. Input form for "Query by decay".

\section{Database Indexes}

An important aspect of the ENSDF database is the publication of evaluations in Nuclear Data Sheets and Nuclear Physics A. An index is provided which shows journal information, authors, and literature cutoff dates for the evaluations contained in ENSDF. This information is extracted from "Comments" datasets. Links to the Nuclear Science References (NSR) database are provided to retrieve references added since the cutoff date.

For selected mass chains, pre-publication datasets are also available via the index page, allowing earlier access to some evaluations.

For the XUNDL database, an index page provides access to the datasets by mass. For each mass, the nuclides covered in the database are shown.

\section{DATASET DISPLAY}

A standard form is used to display the lists of datasets resulting from the various queries. Using this form, the user can select which datasets to display or download. An example is shown in Fig. 3. The ordering of the datasets is based on nuclide, dataset type, and information contained in the DSID field. If the list contains datasets from both ENSDF and XUNDL, the datasets are listed seperately, as shown in the figure. 


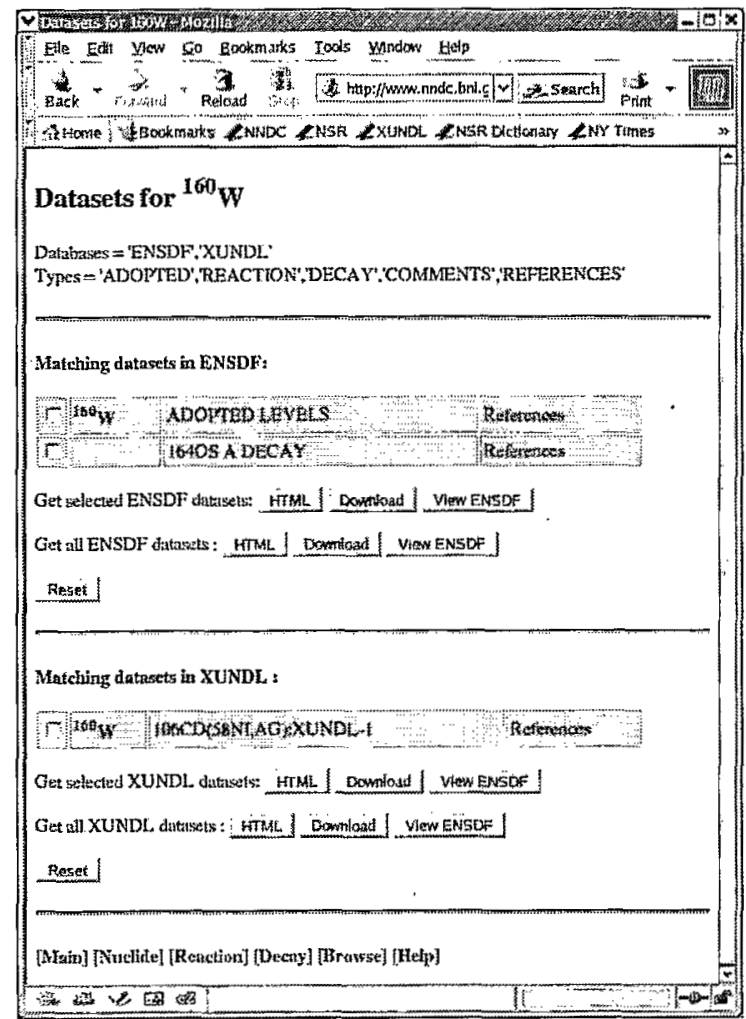

FIGURE 3. Standard output form for query results.

Note that information on the query parameters is displayed at the top, followed by a list of datasets found, grouped by database. Checkboxes to the left of each dataset allow the user to choose particular datasets of interest, and the buttons below the list are used for dataset retrieval. The "HTML" option uses the same WEBTREND application available in earlier versions of ENSDF web access. The "Download" option can be be used if further processing of the dataset is to take place. A portion of the WEBTREND output for the ${ }^{160} \mathrm{~W}$ XUNDL dataset is shown in Fig. 4. This output includes options for the generation of level schemes and band drawings in various formats.

\section{CROSS-LINKING ENSDF AND NSR}

Historically, all references cited in ENSDF are entered into the NSR database and assigned "keynumbers". These keynumbers can then be used to retrieve reference information directly from the NSR database.

On the web form shown in Fig. 3, choosing the "References" link to the right of the dataset specification will bring up a list of all references, extracted from NSR based on the keynumbers used in the dataset. The inverse function is also available: when a list of references

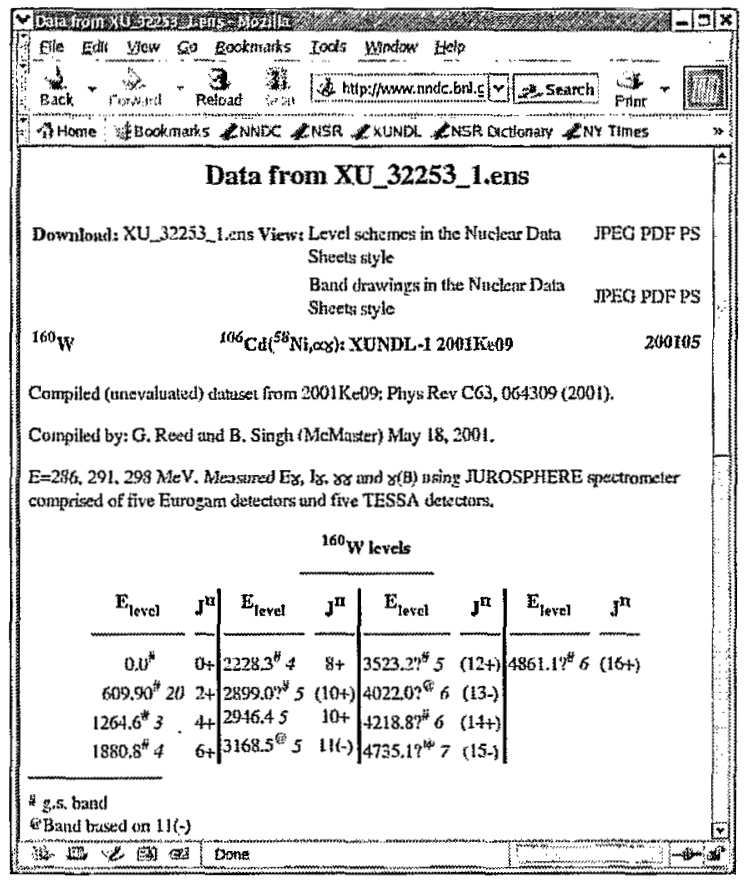

FIGURE 4. Partial WEBTREND output for an XUNDL dataset.

is produced, it includes links to retrieve all datasets citing a given article.

As mentioned in an earlier section, NSR can also be used from the "ENSDF Index" pages to retrieve recent references for a given mass or nuclide.

Within the NSR web application, links to the ENSDF/XUNDL database are provided, where appropriate. For instance, references containing data that has been compiled in XUNDL link to those datasets using a standard form like the one shown in Fig. 3. For NSR entries corresponding to evaluations published in Nuclear Data Sheets, links are provided to the relevant "ENSDF Index" page.

\section{USAGE STATISTICS}

Since the new NNDC web portal went public in April, 2004, the ENSDF/XUNDL web application has received an average of about 230 queries a day, where "query" is defined as a action that retrieves a list of datasets. (This number, and all following, exclude retrievals by known crawlers such as googlebot.com). In the following, usage statistics collected in the four-month period between May 1 and August 312004 are considered.

Over all, there were 25628 queries made during this period, coming from 2485 distinct ip addresses.

Fig. 5 shows the distribution of queries by time of day. It is a fairly wide distribution, with a distinct maximum 


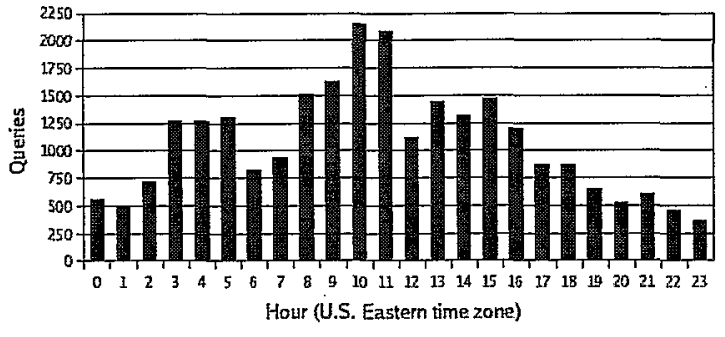

FIGURE 5. Distribution of queries to the ENSDF/XUNDL database, by hour of day.

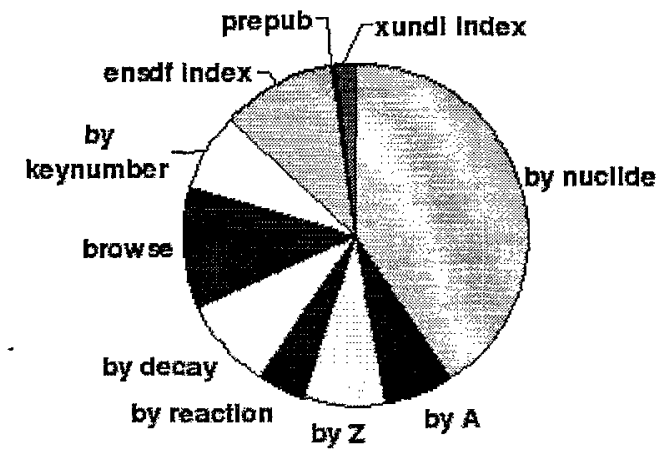

FIGURE 6. Types of queries made to the ENSDF/XUNDL database.

during the morning hours in the eastern United States.

For guiding future development, the type of query being used is of particular interest. In Fig. 6 the relative distribution of query types is shown. By far, the most commonly used approach is to look for datasets corresponding to a particular nuclide (about 4 out of 10 queries). Note that this refers only to nuclide queries from the "Nuclide, Z, A" form; to some extent the browse and index options could be characterized as "by nuclide" as well. In any case, there seems to be enough usage of all the query types to indicate that having a variety of options is worthwhile.

\section{SUMMARY}

The new ENSDF/XUNDL web interface and its underlying data structure were designed and implemented to provide the end user with fast and flexible access to nuclear structure datasets. As more user feedback is received, we hope to continue improving the interface.

It should be noted that, while web access was one of the goals in database design, the same database structure is being used for day-to-day administration of ENSDF and XUNDL, and for generation of publications such as
Nuclear Data Sheets. For the most part, adminstration programs were developed in tandem with the code used in the web application, and many legacy programs were modified to use the new database software.

\section{ACKNOWLEDGMENTS}

The ENSDF/XUNDL database and web design were carried out in collaboration with J.Tuli, T.Burrows, and A.Sonzogni. The National Nuclear Data Center is funded by the Office of Nuclear Physics, Office of Science of the U.S. Department of Energy under Contract No. DE-AC02-98CH10886 with Brookhaven Science Associates, LLC.

\section{REFERENCES}

1. J.K. Tuli, Evaluated Nuclear Structure Data File, BNL-NCS-51655-01/02-Rev (2001) 


\section{DISCLAIMER}

This report was prepared as an account of work sponsored by an agency of the United States Government. Neither the United States Government nor any agency thereof, nor any of their employees, nor any of their contractors, subcontractors, or their employees, makes any warranty, express or implied, or assumes any legal liability or responsibility for the accuracy, completeness, or any third party's use or the results of such use of any information, apparatus, product, or process disclosed, or represents that its use would not infringe privately owned rights. Reference herein to any specific commercial product, process, or service by trade name, trademark, manufacturer, or otherwise, does not necessarily constitute or imply its endorsement, recommendation, or favoring by the United States Government or any agency thereof or its contractors or subcontractors. The views and opinions of authors expressed herein do not necessarily state or reflect those of the United States Government or any agency thereof. 